\title{
Puberty-Defining Normal and Understanding Abnormal
}

\author{
Nirupama K. De Silva, $M D^{1,2, *}$ \\ Jenette Tschirhart, $M D^{2}$
}

\author{
Address \\ ${ }^{1}$ Pediatric and Adolescent Gynecology, University of Oklahoma-Tulsa, Tulsa, OK, \\ USA \\ ${ }^{*}, 2$ Department of Obstetrics and Gynecology, University of Oklahoma-Tulsa, 4502 \\ E 41 st Street, Tulsa, OK, 74135, USA \\ Email: nirupama-desilva@ouhsc.edu
}

Published online: 4 August 2016

C Springer International Publishing AG 2016

\section{This article is part of the Topical Collection on Pediatric Gynecology}

Keywords Puberty $\cdot$ Thelarche $\cdot$ Adrenarche $\cdot$ Menarche Precocious puberty $\cdot$ Delayed puberty

\section{Opinion statement}

Puberty encompasses a multitude of changes as a child transitions to adolescence and adulthood. Over the years, the timing of puberty has seemed to move towards an earlier age than in the past. While genetics plays a role in the timing of puberty, other influences of puberty include race, nutritional status, exposure to endocrine disruptive chemicals, geographic location, psychological factors, and hormonal influences such as leptin and kisspeptin. The typical spectrum of puberty encompasses changes in height, thelarche, a growth spurt, adrenarche, and then menarche. Alterations in the timing of these changes can result in concern and stress for the patient and their family. Prompt attention and careful evaluation for the presence or absence of abnormal puberty is warranted to accurately determine etiology and proper treatment and to prevent long-term sequelae. Consultation with an experienced practitioner can be useful for providers who are not as intimately familiar with the changes of puberty. Once the diagnosis is made, treatment of idiopathic central precocious puberty (CPP) should be accomplished with a GnRH agonist, most commonly leuprolide. Peripheral precocious puberty (PPP) should focus on removing or suppressing the estrogen source, with medication or surgery. When delayed puberty is the source of the problem, treatment is based on finding and treating the underlying cause.

\section{Introduction}

Puberty is a time of transition and growth from childhood to adulthood with the end result being the appearance of adult features and secondary sexual characteristics and the ability to reproduce.
Puberty is initiated upon activation of the hypothalamic-pituitary-ovarian (HPO) axis containing the GnRH pulse generator or gonadostat. Initial activation occurs with increased pulsatile secretion during 
sleep, gradually progressing to secretion throughout the 24-h day [1].

Whether the timing of puberty has changed over the years is a source of much debate amongst researchers, but the most recent data noted from a panel of experts shows that there is an earlier age of breast development and menarchal onset over the last 50 years [2]. Specifically, the Pediatric Research in Office (PROS) data reported that over a cross section of the population, nearly $30 \%$ of the African-American (AA) girls had evidence of breast and/or pubic hair by age 7 and $50 \%$ by age 8 . For Caucasian girls, $15 \%$ had started puberty by age 8 and $40 \%$ by age 9 , with the mean ages for breast and pubic hair being 10 and 10.5 years for Caucasians and 8.9 and 8.8 years for African American girls, respectively. The average age of menarche was 12.16 for AA females and 12.8 for Caucasians [3].

While it does appear that puberty is happening earlier in females, we do not know the exact mechanism, though experts suspect a variety of influences. We do know that over half of pubertal timing is considered heritable [4]. Other influences of puberty include race [4], nutritional status, exposure to endocrine-disruptive chemicals [2], geographic location, psychologic factors, and hormonal influences such as leptin [1]. Leptin, a hormone secreted by adipose tissue, is thought to be a factor involved in puberty. In mice, it has been noted that leptin's influence on adipose tissue affects the timing of puberty [5]. It has also been suggested that the plasma level of kisspeptin is associated with the initiation of pubertal development, and kisspeptin may serve as an important factor in the diagnosis of abnormal puberty [6].

\section{Physical signs of puberty}

Puberty is a continuum of events with wide variations amongst individuals. The first somatic sign of puberty is an increase in growth velocity [4]. This change in height can often go unnoticed by the untrained eye, so in females, most people identify the formation of breast buds as the first visible sign of puberty. Most commonly, this occurs between the ages of 8 and 12 years [7].

Next is the appearance of pubic hair (adrenarche), which occurs due to maturation of the adrenal cortex. Like breast development, pubic hair development is described by sexual maturity rating stages with initial hair growth beginning between ages 9 and 13 .

Subsequent to breast and sexual hair development is the growth spurt or maximum growth velocity. In girls, the growth spurt ceases at a bone age of approximately 15 years with closure of the epiphyseal plates. Puberty also results in significant weight gain, as $50 \%$ of adult body weight is gained in adolescence. In girls, weight gain occurs approximately 6 months after their growth spurt [8].

Menarche, the onset of the menstrual period, usually occurs as the last physical sign of puberty. The age of menarche varies greatly in the USA with the average age being 12.8 [1] (Fig. 1). Less visible changes of puberty include enlargement of the uterus, ovaries, labia, and clitoris.

\section{Evaluation for abnormal pubertal changes}

Initial evaluation of suspected precocious puberty should begin with thorough history and physical exam. The overall timing of pubertal changes should also be kept in mind. Patient and parents should be asked about symptoms such as abdominal pain or urinary and bowel changes, which can be seen in a patient with an abdominal mass. Additional symptoms to inquire about include a history of chronic disease or illness, an evaluation for the presence of stressors, a history of previous chemotherapy/radiation or previous surgery, neurologic symptoms, current medicines, and a history of substance abuse and/or abnormal eating patterns [7]. Family history of development of puberty, disorders such as adultonset congenital adrenal hyperplasia, and patient history of CNS damage should be elicited. Possible exposure to exogenous estrogen should also be discussed with patient and family.

Physical exam should include patient's current height, weight and body mass index, as well as evaluation on the growth curve to determine presence of increased growth velocity. Blood pressure and vital signs should be monitored. Physical exam should include viewing the eye for evidence of papilledema resulting from intracranial masses, evaluation of a patient's smell if any concern, and exam of the thyroid for evidence of goiter or hypothyroidism. Signs of virilization such as deepening of the voice, clitoromegaly, or hirsutism should prompt evaluation for causes of androgen excess. An exam of the breast tissue and external genitalia should be performed with attention to 


\section{Normal Development}

\begin{tabular}{lllcc} 
Growth Spurt & Thelarche & Adrenarche & Peak Height Velocity & Menarche \\
\hline 9.6 yoa & 10.9 yoa & 11.2 yoa & 11.5 yoa & 12.7 yoa
\end{tabular}

Fig. 1. Sequence of events in normal pubertal development.

sexual maturity ratings to evaluate for evidence of advanced maturity. An internal vaginal exam is not usually required. If an evaluation of the internal pelvis is felt to be necessary, a rectal exam is often tolerated better than a pelvic exam in a young child. Alternatively, to avoid an exam, imaging can support your evaluation.

Laboratory testing that may promote insight into the diagnosis includes a thyroid-stimulating hormone, follicle-stimulating hormone (FSH), luteinizing hormone (LH), estradiol, and prolactin. Labs such as DHEA-S and 17-OH progesterone assess a possible androgen source and help evaluate for disorders such as mild or non-classical congenital adrenal hyperplasia [7]. Hormone levels in the pubertal range in a very young patient give concern for abnormal puberty. If LH and FSH results are equivocal or concerning for precocious puberty, a GnRH stimulation test using $100 \mu \mathrm{g}$ of GnRH with subsequent lab draw after 30 min resulting in elevated LH and FSH levels indicates CPP.

When an ultrasound is needed to evaluate the pelvic organs, consider sending the patient to a facility that is accustomed to the pediatric population, so that optimal images can be obtained [7]. Studies have shown that ultrasound examination of the uterus and ovaries served as a complementary tool for the diagnosis of abnormal puberty and thus treatment of the problem [9].

Additional imaging includes an assessment of the bone age of the left hand via plain x-ray to determine the degree of advancement or delay and to help predict final adult height. [10]
History, exam, labs, and bone age together will often help differentiate normal from abnormal puberty. Based on the initial evaluation, one can determine if additional imaging is needed. MRI imaging of the head has the highest sensitivity for detecting masses related to the pituitary and hypothalamic regions but it is not warranted for all patients with a suspicion for abnormal puberty. Rather, recent data reports that it is unclear if girls older than 6 years require MRI in the absence of a suspicion for an intracranial mass [11]. As noted earlier, ovaries can be imaged with ultrasound to rule out an adnexal mass, and imaging of the adrenal glands can be accomplished with CT.

\section{Isolated forms of early puberty}

Premature thelarche Premature thelarche (PT) consists of isolated breast tissue development which can be seen at an early age in a patient who shows no additional signs of precocious puberty. The typical presentation of PT is the appearance of glandular tissue in girls age 2 or younger, with little increase over the period of many months [12]. While some feel PT can be managed without hormonal testing or imaging in most girls without continued breast development and/or rapid growth velocity [12], given that a small percentage of these patients do progress to true precocious puberty, others feel that an initial hormone workup and imaging for abnormal puberty is warranted (as PT can be hard to distinguish from true precocious puberty). According to the latter school of thought, serial measurements of pubertal changes, growth acceleration, hormone labs to evaluate 
for abnormal puberty, and bone age should be undertaken to evaluate for those patients that will actually progress to true precocious puberty [10].

Premature adrenarche Premature adrenarche (PA) occurs due to maturation of the adrenal gland and is associated with the onset of pubic hair. When this event occurs before the age of 8 in girls but is associated with a normal rate of growth and no evidence of clitoromegaly, the diagnosis of premature adrenarche can be made. This diagnosis is more common in African-American girls given their earlier onset of pubic hair [12].

As the adrenal gland is activated without activity from the hypothalamic-pituitary-ovarian (HPO) axis, typical laboratory findings include an elevated DHEAS but prepubertal FSH, LH, and estradiol concentration. The use of bone age has been found to be controversial given the fact that interpretation can be variable when comparing the readings of radiologists to endocrinologists. Further it has been found that $30 \%$ of patients with PA had advanced bone ages and that this had a low predictive value for pathology [13]. Adrenal tumors, late-onset congenital adrenal hyperplasia (via measurement of 17-OHP), exogenous androgen exposure, and true precocious puberty must be eliminated as possible causes before final diagnosis of premature adrenarche can be made. Isolated premature adrenarche has been associated with hyperandrogenism, decreased ovulatory function, polycystic ovarian syndrome, hyperinsulinemia, and elevated triglyceride levels in adulthood. Therefore, for patients with PA, monitoring for these conditions as they progress through adolescence into adulthood is warranted [8]. Concerning cases may warrant referral to a pediatric specialist with expertise in this area.

\section{Central and peripheral forms of precocious puberty} Precocious puberty (PP) occurs more often in girls and can occur at any point in childhood. The lower limit for normal onset of puberty is considered 8 years old. The onset of pubertal events prior to 6 years of age certainly warrants evaluation, but between ages $6-8$, a cross section of females may be experiencing normal puberty. While this may represent normal development in this age group, most providers recommend evaluation for PP, given the anxiety and stress that may be incurred by patients and parents in these situations [1]. Further, timely treatment of precocious puberty is extremely important as precocious physical changes result in increased psychologic stress for a child. Children with precocious puberty may undergo teasing from peers and have an increased risk of sexual abuse and earlier age of coitarche. Last, early fusing of bone plates is promoted by early estrogen secretion and promotes physical changes such as shorter adult stature, which can be traumatic even into adulthood.

Once an evaluation of abnormal puberty is complete, a delineation between normal puberty versus precocious puberty can be made. If there is concern for precocious puberty, the provider must then distinguish if the early puberty is due to a central etiology, known as central precocious puberty (CPP), or a peripheral cause, known as peripheral precocious puberty (PPP).

Central precocious puberty CPP is most often idiopathic and represents premature activation of the HPO axis. Aside from being psychologically and emotionally damaging to a young patient and her family to go through puberty early, the premature secretion of estradiol increases the growth rate and accelerates bone maturation, which can shorten the adult height.

A minority of CPP cases are due to thyroid abnormalities or brain masses, causing direct stimulation of the hypothalamus or pituitary. Masses associated with precocious puberty include pituitary hamartomas, craniopharyngiomas, gliomas, adenomas, and arachnoid cysts. GnRH release can also occur due to hydrocephalus, brain abscess, sarcoidosis, tuberculosis, trauma, radiation, and chemotherapy. Preferred therapy for treatment of both CNS lesions and idiopathic CPP is medical when possible. Surgical therapy is not preferred due to difficulty of removing non-pedunculated lesions without sacrificing normal tissue.

For CPP, treatment with gonadotropin-releasing analog (GnRHA) prevents the secretion of estradiol, slows bone progression, and preserves growth potential. Continuous dosing of GnRH agonists, most commonly leuprolide, causes suppression of the pulsatile GnRH signals from the hypothalamus and promotes children reaching their full heights based on genetics [14]. Giabicani et al. have established formulas to predict adult height to determine if and when treatment with GnRHa is needed [15]. 
Leuprolide can be given in monthly or every 3month injections. Dosing is adjusted until adequate down-regulation of GnRH secretion is obtained by GnRH stimulation test. Low serum concentrations of LH and estradiol, noted via blood samples, and monitoring estradiol and LH responses to leuprolide stimulation can accurately predict pubertal progression [16]. Tests should be repeated every 6-12 months with repeat bone age films every 6 months. Newer options to downregulate GnRH secretion include the 1-year histrelin implant, which also shows good efficacy in treating indiopathic CPP [17]. If serial monitoring of height velocity shows projected shortened adult height, growth hormone administered concurrently with GnRH may improve final adult height.

Traditionally, treatment for CPP is continued until the patient is an optimal height as well as an appropriate emotional and chronologic age to begin puberty and menstruation. Whether height or emotional factors should play a larger role as to the optimal timing of stopping treatments is controversial. Often treatment is discontinued around age 11, but the timing of this should be individualized based on the needs of the patient. Most girls resume menses within 1-2 years of cessation of GnRH treatment.

Peripheral precocious puberty PPP differs from CPP because it does not represent activation of the HPO axis but instead represents a bypass of the hypothalamus and pituitary due to anomalous estrogen production. This estrogen can be produced due to the presence of tumors of the ovary, tumors of the adrenal glands, or from exogenous or endogenous estrogen sources.

McCune-Albright syndrome (MAS) is classically characterized by the triad of café au lait spots on the skin, polyostotic fibrous dysplasia of the bone, and excessive endogenous ovarian estrogen secretion leading to precocious puberty. The disease results from a mutation of the GNAS gene [18]. The diagnosis is a clinical one, and the differential can include CPP, ovarian neoplasm, neurofibromatosis, osteofibrosis dysplasia, and non-ossifying fibromas.

PPP is treated with removal of the estrogen source. In cases of exogenous estrogen (e.g., consumption of birth control pills), removal of exogenous estrogen results in cessation of precocious puberty. In the case of ovarian or adrenal tumors, removal of the tumors results in regression to prepubertal appearance. Patients with PPP from
McCune-Albright are most commonly treated with aromatase inhibitors such as letrazole. More recently, the estrogen antagonist/agonist, tamoxifen, has also shown promise [18]. Secondary CPP in children with MAS is commonly seen after a period of PPP and this is diagnosed by the appearance of advancing puberty in a patient who is well controlled on her medical treatment [18]. In such cases, treatment is with GnRha as for those patients with CPP. Concomitant treatment of fibrous dysplasia should be in conjunction with an expert in that field. Evaluation for concomitant hyperthyroid disease, renal involvement, growth hormone excess, parathyroid abnormalities, or adrenal concerns should be monitored and treated by the appropriate subspecialist.

\section{Delayed puberty}

Unlike early puberty, the timing of delayed puberty has not changed over the years. The absence of thelarche by age 13 and the absence of menses by age 15 or 16 are both considered abnormal and warrant evaluation [4]. According to the American Academy of Pediatrics and the American Congress of Obstetricians and Gynecologists, reasons to evaluate for delayed puberty include [19]

- No thelarche by age 13 years

- Absence of menarche by age 14 years in the presence of hirsutism, history of an eating disorder, or excessive exercise or concern for an outflow abnormality

- Absence of menarche by age 15 years

Further, if there is ever a concern that the continuum of puberty is not being followed appropriately, evaluation for abnormal puberty is warranted [20].

\section{Possible etiologies of delayed puberty}

Two large studies evaluated common causes of delayed puberty in girls. The most common causes of delayed puberty included ovarian insufficiency, permanent hypogonadotropic hypogonadism, constitutional delay of puberty, and congenital absence of the uterus and vagina $[21,22]$. While these are the most common causes of delay, many other causes are also possible (Table 1).

Evaluation for these disorders should start with physical exam (as noted previously) to evaluate for evidence of abnormal or delayed puberty. Laboratory tests can assist in the diagnosis, and possible etiologies can be targeted based on the FSH value. An elevated FSH value should be 


\section{Table 1. Common causes of delayed puberty}

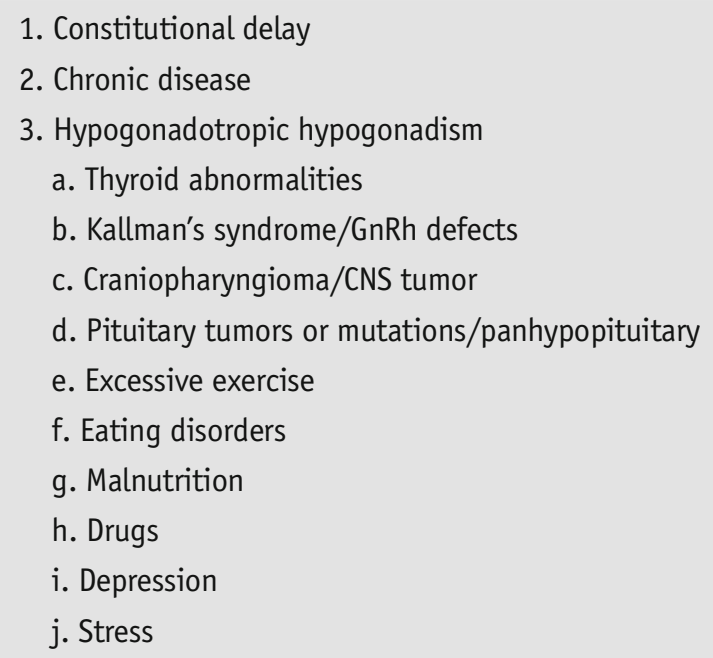

4. Hypergonadotropic hypogonadism
a. Idiopathic premature ovarian insufficiency
b. Fragile $\mathrm{X}$ carrier
c. Turner syndrome
d. Pure gonadal dysgenesis
e. 17 hydroxylase deficiency/aromatase deficiency
f. Galactosemia
g. Sickle cell disease
h. Chemotherapy/radiation
i. Oophorectomy

*From De Silva, NK. Hormonal causes of delayed puberty (permission to use table requested)

repeated to ensure accuracy of the value before the diagnosis of premature ovarian insufficiency (POI) is made. A thorough evaluation for potential causes of delayed puberty is warranted, as $2 / 3$ of all females presenting with delayed puberty have an underlying diagnosis [4]. The most common causes are discussed below [7].

Common causes of ovarian insufficiency or hypergonadotropic hypogonadism An elevated FSH is indicative of premature ovarian insufficiency (POI). As there are multiple causes of POI at a young age, a workup is warranted. POI may be due conditions such as galactosemia (Guerrero) or sickle cell disease as it has been noted that $20 \%$ of patients with sickle cell disease have delayed puberty [1]. A history of radiation, chemotherapy, or oophorectomy will also result in an elevation of gonadotropins. Further, genetic disorders, such as a fragile $X$ mutation or Turner syndrome may cause this clinical picture [7]. Finally, $50 \%$ of teenage patients with primary amenorrhea and no associated comorbidities are found to have an abnormal karyotype, while $13 \%$ of patients with secondary amenorrhea have also been noted to have an abnormal karyotype [23]. Thus, once a diagnosis of POI is made, a chromosome analysis is recommended.

Chromosomal abnormalities A karyotype of $45 \times 0$ reveals the diagnosis of Turner syndrome, and an evaluation for comorbidities of this disorder should be ordered. If elements of a $Y$ chromosome are seen within the genetic make-up, gonadal excision is required, due to the $20-30 \%$ risk of malignant transformation [10]. In patients with Turner 
syndrome, after growth has been optimized with growth hormone, pubertal progression can ensue if in keeping with the patient's social maturity [24].

Pure gonadal dysgenesis is defined as a person with normal stature, streak gonads, and an elevated FSH. The term "pure" refers to the fact that there are no dysmorphic features and the karyotype is either $46 X X$ or XY. With a 46XY karyotype, the diagnosis is Swyer's syndrome. In this disorder, the streak ovaries do not produce androgens or anti-Müllerian hormone, and so the Müllerian system develops in utero by default and without complication. Such patients require gonadal excision due to the risk of malignancy [10].

In $6 \%$ of females with POI and a normal karyotype, a premutation in the FMR1 gene, the gene that promotes the syndrome of fragile $X$, is present [23]. Approximately $1 \%$ of premutation carriers will experience menstrual cessation prior to 18 years of age [25]. Thus, testing for fragile $X$ is warranted for young women with elevated follicle-stimulating hormone (FSH) levels and a normal karyotype, especially if there is a family history of either POI, fragile $X$ syndrome, or undiagnosed mental delays.

Chemotherapy/radiation Chemotherapy and/or radiation can affect a patient's normal pubertal development. With chemotherapy, the type of medication(s) received, the number of doses given, and the age of the patient at time of treatment all have an impact on gonadotoxicity. The highest incidence of acute ovarian failure (AOF) occurs after the use of alkylating agents or procarbazine, but the younger the patient's age during the treatment, the more likely that some ovarian follicles will survive [26].

Whole body, brain, pelvic and/or spinal radiation also increase the risk of AOF [27]. Pelvic radiation doses are directly proportional to AOF and studies reveal that adolescents receiving over 10 Gy acquire acute failure [26]. Chemotherapy with radiation is additive and further promotes the chance of AOF. Thus, in these patients, while spontaneous menses is reassuring, even females who start their menses have an increased lifetime risk of POI [7, 27]

Common causes of hypogonadotropic hypogonadism A low FSH insinuates a different set of disorders in the patient with delayed puberty. Patients with an endocrinopathy, such as a thyroid abnormality, a CNS disorder, chronic disease, poor nutrition, and/or extreme exercise can result in delayed puberty due to low gonadotropin levels. Further, tumors and exposure to certain drugs are other possible etiologies of delayed puberty.
Tumors of the brain/CNS disorders When evaluating a patient for CNS disorders, labs should evaluate for prolactin elevation, while imaging should look for pituitary abnormalities, other brain tumors, and/or a lack of olfactory bulbs. The most common neoplasm of the brain that is associated with pubertal delay is the craniopharyngioma, with a peak incidence between ages 6 and 14. The tumor can be seen on imaging and treatment is surgical, with the possible addition of radiation [1]. Kallman's syndrome is an illness caused by a deficiency in GnRH production and is associated with delayed puberty and anosmia. Anosmia, the lack of smell, is due to a lack of full development of the olfactory bulbs and is the hallmark of this disease.

Chronic disease Chronic illness can cause growth delays and/ or pubertal delays. The severity of the delay is based on personal factors, such as the time of onset of the illness and the severity of the disease. Potential exacerbating factors to the patient's underlying disease include recurrent illnesses/ infections, possible immunodeficiency, and associated emotional stress or due to a disturbance in any major organ that the underlying disorder may affect. Further, poorly controlled illnesses, such as diabetes, hypothyroidism, or Cushing's, can result in pubertal abnormalities.

Malnutrition and the associated inflammatory response caused by poor nutrition can also attribute to delayed puberty. For instance, in children with inflammatory bowel disease, poor nutrition has been shown to affect growth hormone and insulin-like growth factor-1 (IGF-1), which may also alter the growth plates of the long bones. Further, malnutrition results in lower fat mass and decreased leptin levels, which are other possible etiologies for delayed puberty. (Erzi)

Extreme exercise Chronic exercise and/or excessive physical training leads to hypogonadotropic hypogonadism, due to the effect on the HPO axis. Given the need for high energy output in the face of low calorie intake and low body fat, delayed puberty ensues. The lack of fat stores leads to a dearth of leptin, which is postulated to affect puberty [28, 29]. The effects of exercise vary based on the age that the training occurs and the intensity of the training. The groups at highest risk for delayed puberty are those with a lean physique and also those adolescents who began extreme training at a younger age (gymnasts, ballet dancers, figure skaters, and long-distance runners) [28]. In regards to managing an individual athlete's expectations for puberty, both individually and within a sport, teachers/coaches play an important role [30]. 
Constitutional delay Constitutional delay is one of the most common causes of delayed puberty and accounts for approximately 10-30\% of cases [4]. However, this is a diagnosis of exclusion, and a full workup is warranted to look for other causes of delay before this diagnosis can be entertained.

\section{Treatment for delayed puberty}

Treatment options for delayed puberty are based on the etiology. For instance, a thyroid abnormality should be treated by repleting the appropriate hormone; or if the patient has a diagnosis of malnutrition, the treatment is optimizing diet.

In patients with an estrogen deficiency, the treatment is estrogen add-back. Treatment is usually between 12 and 14 years of age to allow for optimal height to occur (as estrogen ingestion will promote closure of the epiphyseal plates.), but treatment should be individualized based on the patient's needs and personal maturity. Goals include promoting physiologic breast development and initiation of menses, while optimizing height and bone mass. For those patients with Turner syndrome, it is common to give growth hormone prior to hormonal estrogen initiation to ensure optimal linear growth [10].

There are a number of estrogen options available to initiate pubertal development. Traditional oral contraceptive pills are not recommended as the initial medicine to promote pubertal development, as this level of synthetic hormone is not at a physiologic level. Estradiol is preferred to initiate puberty. Though oral estradiol is used by many providers, the transdermal route appears to provide more physiologic levels of estradiol and IGF-1 [10] and promotes faster uterine growth and more optimal bone density in the first year of use [31]. Once initiated, estrogen doses should be increased gradually to promote opti- mal breast growth. The timing of increasing doses must be individualized based on the bone age, the predicted height, and the desired timeframe of breast development [10]. Optimally, menarche will be initiated with progesterone use but only after full breast growth has been noted. While there are many options and medical regimens to promote puberty, common options are noted below $[1,7]$ :

(a) Initiate $0.3-\mathrm{mg}$ conjugated estrogen or $0.5-\mathrm{mg}$ micronized estradiol daily. After 6 months to a year, move to a monthly program with $0.625-\mathrm{mg}$ conjugated estrogen or 1.0-mg estradiol and 5mg medroxyprogesterone acetate for the first 14 days of each month.

(b) Start transdermal estrogen patch at 6.5-12 $\mu \mathrm{g}$ (patches can be cut into $1 / 2$ or $1 / 4$ segments to start) and gradually increase the dose over 12 24 months. Once breast development has started and the transdermal estrogen has been increased to a $50-\mu \mathrm{g}$ patch, or menses spontaneously occurs, begin progesterone add-back. A short course of medroxyprogesterone or micronized progesterone given at the same time monthly is optimal for patients so that they can have predictable cycles.

After pubertal growth is complete, long-term maintenance requires the cyclic use of an estrogen and a progesterone. At this point, either oral or transdermal estrogen in conjunction with cyclic progesterone or an oral contraceptive pill are both reasonable options. Of note, patients with POI require prolonged treatment, while those with constitutional delay may be able to stop when pubertal development is complete.

\section{Conclusion}

In conclusion, puberty is a spectrum and prompt attention and careful evaluation for the presence or absence of abnormal puberty is warranted to accurately determine etiology and proper treatment and to prevent long-term sequelae. Consultation with an experienced practitioner can be useful for providers who are not as intimately familiar with the changes of puberty. 


\section{Compliance with Ethical Standards}

\section{Conflict of Interest}

Nirupama K. De Silva and Jenette Tschirhart declare that they have no conflict of interest.

Human and Animal Rights and Informed Consent

This article does not contain any studies with human or animal subjects performed by any of the authors.

\section{References and Recommended Reading}

1. Speroff L, Glass RH, Kase N. Clinical gynecologic endocrinology and infertility. 6th Ed. Baltimore: Lippincott Williams and Wilkins; 2005. Chapter 10, Abnormal Puberty and Growth Patterns, pg 361-400.

2. Euling SY, Selevan SG, Pescovitz OH, Skakkebaek NE. Role of environmental factors in the timing of puberty. Pediatrics. 2008;121:S167.

3. Herman-Giddens ME, Slora EJ, Wasserman RC, Bourdony CJ, Bhapkar MV, Koch GG, et al. Secondary sexual characteristics and menses in young girls seen in the office practice: a study from the Pediatric Research in Office Settings network. Pediatrics. 1997;99(4):50512.

4. McLean M, Davis AJ, Reindollare RH. Abnormalities of female pubertal development. MedText.com 2015.

5. Bohlen TM, Silveira MA, Zampieri TT, Frazao R. Fatness rather than leptin sensitivity determines the timing of puberty in female mice. Mol Cell Endocrinol. 2016;423:11-21.

6. Yang YU, Xiong XY, Yang LI, Xie L, Huang H. Testing of kisspeptin levels in girls with idiopathic central precocious puberty and its significance. Exp Ther Med. 2015;9:2369-73.

7. De Silva NK. Hormonal causes of delayed puberty, In: Female Puberty: A comprehensive Guide for Clinicians, Dietrich J(Ed), Springer Science, 2014 Aug.

8. Alvero, R, Schlaff WD. Reproductive endocrinology and infertility: the requisites and obstetrics and gynecology 1st Ed. 2007 Chapter 3, Normal and Abnormal Puberty; p 33-48.

9. Badouraki M, Christoforidis A. Economou et al. Evaluation of pelvic ultrasonography in the diagnosis and differentiation of various forms of sexual precocity in girls. Ultrasound Obstet Gynecol. 2008;32:819-27.

10. Emans SJ, Laufer MR. Pediatric and adolescent gynecology. 6th ed. Philadelphia: Lippincott Williams and Wilkins; 2012. Chapter 7 and 8, Precocious and Delayed Puberty; p 114-37.

11. Pedicelli S, Alessio P, Scire G, et al. Routine screening by brain magnetic resonance imaging is not indicated in every girl with onset of puberty between ages of 6 and 8 years. J Clin Endocrinol Metab.

2014;99(12):4455-61.

12. Kaplowitz P, Bloch C. Evaluation and referral of children with signs of early puberty. American Academy of Pediatrics: Clinical Report 2016.

13. DeSalvo DJ, Mehra R, Vaidyanathan P, Kaplowitz PB. In children with premature adrenarche, bone age advancement by 2 or more years is common and generally benign. J Pediatr Endocrinol Metab. 2013;26(34):215-21.

14. Borges MF, Franciscon PM, Cambraia TC, Oliveira DM, Ferreira BP, Resende EAMR, Palhares HMC. Evaluation of centra; precocious puberty treatment with GnRH analogue at the Triangulo Mineiro Federal University (UFTM). Arch. Endocrinol. Metab. 2015; vol.59 no. 6.

15. Giabicani E, Lemaire P, Brauner R. Models for predicting the adult height and age at first menstruation of girls with idiopathic central precocious puberty. PLoS ONE. 2015;10(4), e0120588.

16. Sathasivam A, Garibaldi L, Shapiro S, Gadbold J, Rapaport R. Leuprolide stimulation testing for the evaluation of early female sexual maturation. Clin Endocrinol. 2010;73:375-81.

17. Rosati SM et al. Histrelin for central precocious puberty-a single surgeon experience. J Surg Res. 2015;198(2):355-9.

18. Dumitrescu CE, Collins MT. McCune-Albright syndrome. Orphaned J Rare Dis. 2008;3:12.

19. ACOG/AAP Statement: The menstrual cycle as a vital sign, 2006

20. American Medical Association. Guidelines for Adolescent Preventive Services (GAPS). [Website]. 1997; http://www.ama-assn.org/ama/pub/physicianresources/public-healthservices.shtml. Accessed Feb 8, 2016.

21. Reindollar RH, Byrd JR, McDonough PG. Delayed sexual development: a study of 252 patients. Am J Obstet Gynecol. 1981;140(4):371-80. 
22. Sedlmeyer IL, Palmert MR. Delayed puberty: analysis of a large case series from an academic center. J Clin Endocrinol Metab. 2002;87(4):1613-20.

23. Nelson LM, Covington SN, Rebar RW. An update: spontaneous premature ovarian failure is not an early menopause. Fertil Steril. 2005;83:1327-32.

24. Bannink EMN, van Sassen C, van Buurent S, de Jong FH, Lequins M, Mulder PGH, et al. Puberty induction in Turner syndrome: results of oestrogen treatment on development of secondary sexual characteristics, uterine dimensions and serum hormone levels. Clin Endocrinol. 2009;70:265-73.

25. De Caro JJ, Dominguez C, Sherman SL. Reproductive health of adolescent girls who carry the FMR1 premutation: expected phenotype based on current knowledge of fragile X-associated primary ovarian insufficiency. Ann N Y Acad Sci. 2008;1135:99-111.

26. Green DM, Sklar CA, Boice Jr JD, Mulvihill JJ, Whitton JA, Stovall M, et al. Ovarian failure and reproductive outcomes after childhood cancer treatment: results from the Childhood Cancer Survivor Study. J Clin Oncol. 2009;27:2374-81.

27. Duffy C, Allen S. Medical and psychosocial aspects of fertility after cancer. Cancer J. 2009;15:27-33.

28. Sanfilippo JS, Lara-Torre E, Edmonds K, Templeman C. Clinical pediatric and adolescent gynecology. 1st Ed. New York: Informa Healthcare USA; 2009. Chapter 4, Delayed Puberty: p 40-61.

29. Fujii K, Demura S. Relationship between change in BMI with age and delayed menarche in female athletes. J Physiol Anthropol Appl Human Sci. 2003;22:97-104.

30. Michell SB, Haase AM, Malina RM. The role of puberty in the making and breaking of young ballet dancers: perspective of dance teachers. J Adolesc. 2016;47:81-9. 31. Nabhan ZM, Dimeglio LA, Qi R, Perkins SM, Eugster EA. Conjugated oral versus transdermal estrogen replacement in girls with Turner syndrome: a pilot comparative study. J Clin Endocrinol Medtab. 2009;94(6):2009-14. 